\title{
Symmetry, Singularities and Integrability in Complex Dynamics V: Complete Symmetry Groups of Certain Relativistic Spherically Symmetric Systems
}

\author{
$P G L L E A C H^{\dagger *}, M C N U C C I^{\ddagger}$ and $S$ COTSAKIS ${ }^{\dagger}$ \\ † GEODYSYC, Department of Mathematics, University of the Aegean, \\ Karlovassi 83 200, Greece \\ * Permanent address: School of Mathematical and Statistical Sciences, \\ University of Natal, Durban 4041, Republic of South Africa \\ $\ddagger$ Dipartimento di Matematica e Informatica, Università di Perugia, 06123 Perugia, Italy \\ Received August 3, 2000; First Revision December 7, 2000; Second Revision May 4, 2001; \\ Accepted May 5, 2001
}

\begin{abstract}
We show that the concept of complete symmetry group introduced by Krause (J. Math. Phys. 35 (1994), 5734-5748) in the context of the Newtonian Kepler problem has wider applicability, extending to the relativistic context of the Einstein equations describing spherically symmetric bodies with certain conformal Killing symmetries. We also provide a simple demonstration of the nonuniqueness of the complete symmetry group.
\end{abstract}

\section{Introduction}

In 1994 Krause [12] introduced the concept of a complete symmetry group in classical mechanics. This concept was somewhat stronger than that of the standard symmetry group generally used at the time, which was the group represented by the Lie point symmetries of the system of differential equations describing the dynamical system. A complete symmetry group realisation in mechanics was required to be endowed with the properties that (i) the group act freely and transitively on the manifold of all allowed motions of the system and (ii) the given equations of motion be the only ordinary differential equations that remain invariant under the specified action of the group. The vehicle which Krause used to demonstrate the concept was the classical Kepler problem described in reduced coordinates by the second order ordinary differential equation

$$
\ddot{\boldsymbol{r}}+\frac{\mu \boldsymbol{r}}{r^{3}}=0,
$$


where $\mu$ is a constant, $\boldsymbol{r}=\left(x_{1}, x_{2}, x_{3}\right)$ and $r=|\boldsymbol{r}|$ in a standard notation. The Lie point symmetries of the system are given by

$$
\begin{aligned}
& G_{1}=x_{2} \partial_{x_{3}}-x_{3} \partial_{x_{2}}, \quad G_{2}=x_{3} \partial_{x_{1}}-x_{1} \partial_{x_{3}}, \quad G_{3}=x_{1} \partial_{x_{2}}-x_{2} \partial_{x_{1}}, \\
& G_{4}=\partial_{t}, \quad G_{5}=3 t \partial_{t}+2 r \partial_{r},
\end{aligned}
$$

where $G_{1}, G_{2}$ and $G_{3}$ are the generators of rotation, $G_{4}$ is the generator of time translations and $G_{5}$ represents rescaling which maps orbits of a given eccentricity into orbits of the same eccentricity and is the basis of Kepler's Third Law because of the happy happenstance that for the Kepler problem (and also the simple harmonic oscillator) the eccentricity does not enter into the relationship between the period of the orbit and the length of the semimajor axis as it does for other periodic orbits [6]. The algebra of the point symmetries is $s o(3) \oplus A_{2}$. In addition to the standard Lie point symmetries Krause determined the three nonlocal symmetries

$$
\begin{gathered}
X_{1}=\left(2 \int x_{1} \mathrm{~d} t\right) \partial_{t}+x_{1} r \partial_{r}, \quad X_{2}=\left(2 \int x_{2} \mathrm{~d} t\right) \partial_{t}+x_{2} r \partial_{r}, \\
X_{3}=\left(2 \int x_{3} \mathrm{~d} t\right) \partial_{t}+x_{3} r \partial_{r}
\end{gathered}
$$

and with these eight symmetries was able to specify the precise form of (1.1) up to the value of the constant $\mu$. That the constant $\mu$ could not be specified is not surprising since it can be removed from the equation (1.1) by a rescaling of the vector $\boldsymbol{r}$.

The work of Krause was and is very interesting. Apart from any other features which are peculiar to the Kepler problem, there was presented the new approach to the calculation of nonlocal symmetries which are not easy to calculate in an algorithmic way [7]. It is unfortunate that in his paper Krause made the comment that the complete symmetry group could not be obtained for the Kepler problem by the standard analysis used for Lie point symmetries. Rising to the implied challenge Nucci [23] proceeded to devise a scheme of reduction of order of the system, in essence a removal of time as the independent variable so that the coefficient function of $\partial_{t}$ appeared only as the derivative and hence a local function for the type of nonlocal symmetry used by Kause, which made the determination of the nonlocal symmetries above a process of the determination of the Lie point symmetries of the reduced system. More recently Nucci and Leach [24] have shown that the number of nonlocal symmetries of this class for the Kepler problem was somewhat greater than Krause had reported. Furthermore, in contrast to his statement that one does not obtain a Lie algebra when the nonlocal symmetries are considered, they presented the algebra.

Krause emphasised that his treatment applied to mechanical systems based on the Newtonian laws, but there can be no doubt that the concept of a complete symmetry group is applicable to all systems of ordinary differential equations which possess a suitable number of symmetries to define precisely the system of equations. Krause further remarks that it is not known whether the specific realisation of a complete symmetry group exists for any given Newtonian system - more generally system of ordinary differential equations and that the question was open. There is a specific realisation for one-dimensional linear second order equations, $\operatorname{sl}(3, R)$, but even for nonlinear one-dimensional systems the problem was unresolved. This immediately raises the question of what the situation would be 
in the case of dynamical systems known to display the characteristics of nonintegrability. Considering that the existence of symmetry is regarded as one of the criteria relating to integrability, the concept of the complete symmetry group of a nonintegrable dynamical system would appear to present something of a paradox.

In this paper, which is the fifth of a series [16, 17, 18, 22] devoted to investigating the connections between the three main topics in dynamics, videlicet symmetry, singularities and integrability, topics which superficially are unrelated in their mathematics and yet are intimately intertwined, we demonstrate that it is possible to have a complete symmetry group of an ordinary differential equation which does not have sufficient Lie point symmetries for integrability and is not integrable in the sense of Painlevé. In the process we demonstrate the resolution of the apparent paradox.

In the normal course of events one associates the existence of symmetry in a differential equation (or system of differential equations) with the integrability of the equation. However, the normal course of events also restricts the consideration of a symmetry to the class of point, contact or generalised symmetries. In the very introduction of the concept of a complete symmetry group Krause was obliged to resort to nonlocal symmetries for the complete specification of the system of differential equations for the Kepler problem which is well-known to be an integrable system. The need to use nonlocal symmetries for the complete symmetry group is not automatic. For example the equation $y^{\prime \prime}=0$ is specified completely by three point symmetries.

In this paper we are not concerned with the existence of symmetries which will enable the equation to be integrated. We are concerned with the existence of symmetries which will enable the equation to be specified completely, perhaps up to an arbitrary parameter as was the case of Krause's complete symmetry group for the Kepler problem. The role played by the elements of the complete symmetry group is to fix the form of the dynamical equations. Our aim is to demonstrate that this be the case whether the system be integrable or not integrable. As we shall see, the very nature of the symmetries obtained is not likely to cause one to imput integrability of the model equation. It would appear that symmetries can play two roles, one to specify the nature of the equation and the other to provide part of the route to integrability. A priori one cannot expect both roles to the played by the one symmetry.

This paper is structured as follows. In the next section we review the known properties of our model equation. In Section 3 we calculate its symmetries and demonstrate that within the number of these symmetries there is a subset which is the complete symmetry group of the model equation. In Section 4 we conclude with some observations.

\section{Painlevé nonintegrability}

Our model equation is

$$
y^{\prime \prime \prime}+y^{\prime \prime}+y y^{\prime}=0,
$$

where the prime indicates differentiation with respect to the independent variable $x$. Equation (2.1) arises in general relativity. On the assumption of a conformal Killing vector of a particular type for a spherically symmetric shear-free gravitational field Dyer, McVittie and Oattes |4 obtained the third order field equation

$$
\mu^{2} T_{\mu \mu \mu}+\mu(2 m-1) T_{\mu \mu}+\left(m^{2}-2 m+2 T\right) T_{\mu}=0 .
$$


Under the transformation

$$
\mu=\exp \left(\frac{x}{2 m-4}\right), \quad T(\mu)=2(m-2)^{2} y(x)-\frac{1}{2}\left(m^{2}-4 m+3\right)
$$

we obtain (2.1) from (2.2). To determine the leading order behaviour of (2.1) we make the substitution $y=\alpha \chi^{p}$, where $\chi=x-x_{0}$ into (2.1). We find that the first and the third terms are dominant, $\alpha=-12$ and $p=-2$. The substitution of

$$
y=-12 \chi^{-2}+\beta \chi^{r-2}
$$

into the two dominant terms gives the resonances $r=-1,4,6$. We substitute

$$
y=-12 \chi^{-2}+\sum_{i=-1}^{4} a_{i} \chi^{i}
$$

into the full equation (2.1) to see it is compatible at the resonances. We find that

$$
a_{-1}=\frac{12}{5}, \quad a_{0}=\frac{1}{25} \quad \text { and } \quad a_{1}=\frac{1}{125}
$$

for the coefficients up to the first resonance at $r=4$. At the first resonance we have compatibility and, by construction, $a_{2}$ is arbitrary. The next coefficient is given by

$$
a_{3}=\frac{1+1250 a_{2}}{18750} \text {. }
$$

However, at the resonance, $r=6$, the condition of compatibility requires that

$$
a_{2}=-\frac{1}{1250}
$$

i.e. the coefficient $a_{3}$ is zero. Consequently (2.1) fails the Painlevé test. The solution requires logarithmic terms to be introduced into the Laurent expansion and this means that the general solution has infinitely many branches. Infinite branching is strongly connected with nonintegrability and this suggests that (2.1) could exhibit chaos [1] 28, p. 348]]. Analysis of the numerical properties of the equation reveals that there is instability in the numerical solution but not chaos.

Even a cursory examination of (2.1) reveals the existence of a first integral

$$
I\left(y, y^{\prime}, y^{\prime \prime}\right)=y^{\prime \prime}+y^{\prime}+\frac{1}{2} y^{2}
$$

which is associated with the obvious Lie point symmetry of (2.1), videlicet $\partial_{x}$. Instead of viewing (2.9) as a first integral of (2.1) one can consider it as an equation of the second order containing a parameter, $I$, videlicet

$$
y^{\prime \prime}+y^{\prime}+\frac{1}{2} y^{2}-I=0 .
$$

\footnotetext{
${ }^{1}$ The logarithmic singularity of the general solution can be avoided by accepting the constraint, (2.8), on the coefficient $a_{2}$ so that one obtains the series expansion, $y=-12 \chi^{-2}+\frac{12}{5} \chi^{-1}+25+\frac{1}{125} \chi+\frac{1}{1250} \chi^{2}+$ $a_{4} \chi^{4}+\cdots$. This expansion contains only the two free parameters, $x_{0}$ and $a_{4}$ and is interpreted to be the local representation of a particular solution of (2.1) in the sense of Cotsakis and Leach [3, 16]. There is another interpretation which is that this series expansion is the general solution of a second order equation which, in some sense, is an invariant of the third order equation, (2.1).
} 
This equation can be written as a family of two-dimensional first order systems,

$$
\begin{gathered}
\dot{x}=y, \\
\dot{y}=-y-\frac{1}{2} x^{2}+I .
\end{gathered}
$$

The equilibrium points of this system occur only for $I \geq 0$. They are $x= \pm \sqrt{2 I}, y=0$. A simple application of the Bendixon-Dulac criterion reveals that there are no limit cycles. Thus, as $I$ goes from negative to positive values, the system experiences a supercritical bifurcation with an additional qualitative change in the nature of the critical points. The equilibrium points on the positive $x$ axis are always saddle points. On the other hand those lying on the negative $x$ axis are stable nodes for $I<1 / 32$ and stable foci with clockwise direction for $I>1 / 32$.

It is sometimes natural to consider the dynamics of a two-dimensional family of systems depending upon parameters as equivalent to that of a higher order model equation from which it can derived. In our case, however, since the two-dimensional reduced system has bifurcations and lines of equilibria it may have completely different (and simpler) dynamics than that hidden in the third order equation. In fact we can apply the Painlevé test to (2.10) and find that it satisfies the necessary conditions for the possession of the Painlevé property only in the case that $I=-18 / 625$. Then it has the expansion

$$
y=-12 \chi^{-2}+\frac{12}{5} \chi^{-1}+25+\frac{1}{125} \chi-\frac{1}{12500} \chi^{2}-\frac{1}{187500} \chi^{3}+b_{4} \chi^{4}+\cdots,
$$

where $b_{4}$ is the arbitrary constant introduced at the resonance $r=6$. The integrability of

$$
y^{\prime \prime}+y^{\prime}+\frac{1}{2} y^{2}+\frac{18}{625}=0
$$

has been demonstrated by Ince [10] who transformed (2.14) to

$$
\frac{\mathrm{d}^{2} w}{\mathrm{~d} z^{2}}=6 w^{2},
$$

the solution of which can be expressed in terms of elliptic functions, by means of the Kummer-Liouville transformation [13, 20]

$$
y(x)=\exp \left(-\frac{2 z}{5}\right) w(x)+\frac{6}{25}, \quad x=-\frac{5 i}{\sqrt{12}} \exp \left(-\frac{z}{5}\right) .
$$

Equation (2.15) is integrable in the sense of Painleve and the transformation (2.16) is single-valued. Hence (2.14) is integrable. Whatever happens to the solutions of (2.1) which commence at some point not on the surface defined by (2.10) in the phase space with the particular value of $I=-18 / 625$ is not obvious, but those which do are regular and remain on the surface.

As a final note on the properties of our model equation we remark that the equation, (2.14), has the additional Lie point symmetry (calculated using Program Lie [9, 27]), $\exp \left(\frac{x}{5}\right)\left[\partial_{x}-\left(\frac{2}{5} y-\frac{12}{125}\right) \partial_{y}\right]$, in addition to the symmetry, $\partial_{x}$, possessed for general values of the parameter, $I$. The algebra of the symmetries is Type III of Lie's classification of two-dimensional algebras [19, p. 430, Kap. 19] which has the normal form

$$
G_{1}=\partial_{y}, \quad G_{2}=x \partial_{x}+y \partial_{y}
$$


so that $\left[G_{1}, G_{2}\right]=G_{1}$ and the normal form of the equation invariant under the two symmetries (2.17) is

$$
X Y^{\prime \prime}=F\left(Y^{\prime}\right) .
$$

The tidy form of the equation, (2.15), arises from using the nonnormal form

$$
G_{1}=\partial_{x}, \quad G_{2}=-x \partial_{x}+y \partial_{y}
$$

The second symmetry in (2.17) should not be expected to be related to the complete symmetry group to be computed since (2.14) is not equivalent to (2.1) as the particular value of $I$ brings us into the realm of configurational invariants [8, 26].

\section{$3 \quad$ Lie symmetries}

Equation (2.1) has just the one Lie point symmetry, $\partial_{x}$, which cannot be the complete symmetry group of (2.1) since the equation

$$
y^{\prime \prime \prime}=f\left(y, y^{\prime}, y^{\prime \prime}\right)
$$

is the general form of a third order ordinary differential equation invariant under $\partial_{x}{ }^{2}$. Examination of (2.1) for contact symmetries shows that there are none. Consequently the complete symmetry group (we now speak strictly in the sense of Krause) must comprise nonlocal or generalised symmetries. To calculate these we follow the procedure outlined in Pillay et al [25] and used with great effect by Bouquet et al [15] in their study of second order ordinary differential equations possessing integrating factors [2].

When one studies symmetries of differential equations without placing any restrictions upon the coefficient functions one may replace the differential operator

$$
G=\xi \partial_{x}+\eta \partial_{y}
$$

with the equivalent operator

$$
\bar{G}=\bar{\eta} \partial_{y},
$$

where $\bar{\eta}=\eta-y^{\prime} \xi$. The advantage of using (3.3) is that the calculations are simpler. Dropping the overbar we find that the action of the third extension of (3.3) on (2.1) gives

$$
\eta^{\prime \prime \prime}+\eta^{\prime \prime}+\eta^{\prime} y+\eta y^{\prime}=0
$$

Our task is to find a sufficient number of independent functions, $\eta$, which are solutions of (3.4) to be able to define (2.1) uniquely and so constitute the required number of symmetries to make a complete symmetry group.

It is a trivial matter to integrate (3.4) once. We have

$$
\eta^{\prime \prime}+\eta^{\prime}+\eta y=C,
$$

\footnotetext{
${ }^{2}$ One could consider introducing the idea of degrees of completeness in symmetry groups. This would depart from the criterion of Krause that the equation be unique. However, there is no reason why we should not elaborate on Krause's proposition.
} 
where $C$ is an arbitrary constant of integration. We replace the coefficient of $\eta$ from the original differential equation, (2.1), to obtain

$$
\left(\eta^{\prime \prime}+\eta^{\prime}\right) y^{\prime}-\eta\left(y^{\prime \prime \prime}+y^{\prime \prime}\right)=C y^{\prime}
$$

which can be integrated by parts to give

$$
\eta^{\prime} y^{\prime}-\eta y^{\prime \prime}+\int\left(\eta^{\prime} y^{\prime}-\eta y^{\prime \prime}\right) \mathrm{d} x=C y+K
$$

where $K$ is another arbitrary constant. If we let $\omega=\int\left(\eta^{\prime} y^{\prime}-\eta y^{\prime \prime}\right) \mathrm{d} x$, the integrodifferential equation (3.7) becomes the first order linear nonhomogeneous equation

$$
\omega^{\prime}+\omega=C y+K
$$

which has the solution

$$
\omega=J \mathrm{e}^{-x}+\mathrm{e}^{-x} \int(C y+K) \mathrm{e}^{x} \mathrm{~d} x,
$$

where $J$ is a constant of integration, so that $\eta$ now satisfies its own linear first order nonhomogeneous equation,

$$
\eta^{\prime} y^{\prime}-\eta y^{\prime \prime}=C y+K-J \mathrm{e}^{-x}-\mathrm{e}^{-x} \int(C y+K) \mathrm{e}^{x} \mathrm{~d} x
$$

The solution of (3.10) is

$$
\eta=A y^{\prime}+y^{\prime} \int \frac{1}{y^{\prime 2}}\left[C y+K-J \mathrm{e}^{-x}-\mathrm{e}^{-x} \int(C y+K) \mathrm{e}^{x} \mathrm{~d} x\right] \mathrm{d} x,
$$

where $A$ is a constant of integration.

In (3.11) there are four constants, but one of them, $K$, is spurious. We have the three symmetries

$$
\begin{gathered}
G_{1}=y^{\prime} \partial_{y} \Leftrightarrow G_{1}=\partial_{x}, \\
G_{2}=y^{\prime}\left(\int \frac{\mathrm{d} x}{y^{\prime 2} \mathrm{e}^{x}}\right) \partial_{y} \Leftrightarrow G_{2}=\left(\int \frac{\mathrm{d} x}{y^{\prime 2} \mathrm{e}^{x}}\right) \partial_{x}, \\
G_{3}=y^{\prime}\left(\int \frac{\mathrm{d} x}{y^{\prime 2}}\left(y-\mathrm{e}^{-x} \int y \mathrm{e}^{x} \mathrm{~d} x\right)\right) \partial_{y} \\
\Leftrightarrow G_{3}=\left(\int \frac{\mathrm{d} x}{y^{\prime 2}}\left(y-\mathrm{e}^{-x} \int y \mathrm{e}^{x} \mathrm{~d} x\right)\right) \partial_{x},
\end{gathered}
$$

where we have added the corresponding operator when we use only $\partial_{x}$ for the symmetry. We observe that the first of (3.12) is just the obvious symmetry of (2.1).

Our immediate task is to find the form of the third order differential equation which is invariant under the actions of the third extensions of these three symmetries. We commence with the general equation

$$
y^{\prime \prime \prime}=f\left(x, y, y^{\prime}, y^{\prime \prime}\right)
$$


For the calculations we use the $\partial_{x}$ form of the symmetries. Invariance under $G_{1}$ implies that the equation have the form

$$
y^{\prime \prime \prime}=f\left(y, y^{\prime}, y^{\prime \prime}\right) \text {. }
$$

Invariance under $G_{2}$ and $G_{3}$ is not obtainable by inspection! The third extension of $G_{2}$ is

$$
G_{2}^{[3]}=\left(\int \frac{\mathrm{d} x}{y^{\prime 2} \mathrm{e}^{x}}\right) \partial_{x}-\left(\frac{1}{y^{\prime} \mathrm{e}^{x}}\right) \partial_{y^{\prime}}+\left(\frac{1}{y^{\prime} \mathrm{e}^{x}}\right) \partial_{y^{\prime \prime}}-\left[\frac{y^{\prime \prime \prime}+y^{\prime \prime}+y^{\prime}}{y^{\prime 2} \mathrm{e}^{x}}\right] \partial_{y^{\prime \prime \prime}}
$$

and, when it acts on (3.14), we obtain the linear first order partial differential equation

$$
y^{\prime} \frac{\partial f}{\partial y^{\prime \prime}}-y^{\prime} \frac{\partial f}{\partial y^{\prime}}=-f-y^{\prime \prime}-y^{\prime}
$$

which has the two characteristics

$$
u=y^{\prime \prime}+y^{\prime} \quad \text { and } \quad v=\frac{f+y^{\prime}+y^{\prime \prime}}{y^{\prime}}
$$

so that the right side of (3.14) has the form

$$
f=-\left(y^{\prime \prime}+y^{\prime}\right)+y^{\prime} g\left(y^{\prime \prime}+y^{\prime}, y\right)
$$

where $g$ is an arbitrary function of its arguments.

The third extension of $G_{3}$ is

$$
\begin{gathered}
G_{3}^{[3]}=\left(\int \frac{\mathrm{d} x}{y^{\prime 2}}\left(y-\mathrm{e}^{-x} \int y \mathrm{e}^{x} \mathrm{~d} x\right)\right) \partial_{x}-\frac{1}{y^{\prime}}\left(y-\mathrm{e}^{-x} \int y \mathrm{e}^{x} \mathrm{~d} x\right) \partial_{y^{\prime}} \\
-\frac{1}{y^{\prime}}\left(y^{\prime}-y+\mathrm{e}^{-x} \int y \mathrm{e}^{x} \mathrm{~d} x\right) \partial_{y^{\prime \prime}}+\partial_{y^{\prime \prime \prime}} .
\end{gathered}
$$

From the action of (3.19) and (3.14) with $f$ as given in (3.18) we find that $g$ is independent of its first argument and so the general form of the third order equation invariant under the three symmetries $(3.12)$ is

$$
y^{\prime \prime \prime}+y^{\prime \prime}+y^{\prime}+y^{\prime} h(y)=0
$$

where $h(y)$ is an arbitrary function.

It is quite evident from (3.20) that the three symmetries (3.12), which arise from the direct integration of the linear third order ordinary differential equation (3.4), are insufficient to specify completely our model equation, (2.1), and so we must find some other symmetry. We assume that the symmetry has the form $\xi \partial_{x}$. The action of the third extension of the symmetry on (2.1) gives

$$
y^{\prime} \xi^{\prime \prime \prime}+\left(3 y^{\prime \prime}+y^{\prime}\right) \xi^{\prime \prime}+\left(2 y^{\prime \prime \prime}+y^{\prime \prime}\right) \xi^{\prime}=0,
$$

in which (2.1) has been used. Equation (3.21) is exact and can be integrated once to give

$$
y^{\prime} \xi^{\prime \prime}+\left(2 y^{\prime \prime}+y^{\prime}\right) \xi^{\prime}=A,
$$


where $A$ is a constant of integration, which is a linear first order nonhomogeneous equation for $\xi^{\prime}$ with the solution

$$
\xi^{\prime}=A \frac{\int y^{\prime} \mathrm{e}^{x} \mathrm{~d} x}{y^{\prime 2} \mathrm{e}^{x}}+B \frac{1}{y^{\prime 2} \mathrm{e}^{x}}
$$

and so

$$
\xi=A \int\left(\frac{\int y^{\prime} \mathrm{e}^{x} \mathrm{~d} x}{y^{\prime 2} \mathrm{e}^{x}}\right) \mathrm{d} x+B \int \frac{\mathrm{d} x}{y^{\prime 2} \mathrm{e}^{x}}+C,
$$

where $B$ and $C$ are further constants of integration. Unfortunately, and this should not be surprising, the three symmetries in (3.24) are just the three symmetries listed in (3.12).

Evidently we are not going to be able to obtain the necessary additional symmetries the easy way and so we must assume that the symmetry has the form (cf the Ansatz of Krause [12])

$$
G=\left(\int \xi \mathrm{d} x\right) \partial_{x}+\eta \partial_{y}
$$

The action of the third extension of (3.25) on the equation (2.1) gives

$$
y^{\prime} \xi^{\prime \prime}+y^{\prime \prime} \xi^{\prime}+\left(2 y^{\prime \prime}+y^{\prime}\right) \xi^{\prime}+\left(2 y^{\prime \prime \prime}+y^{\prime \prime}\right) \xi=\eta^{\prime \prime \prime}+\eta^{\prime \prime}+\eta^{\prime} y+\eta y^{\prime}
$$

which is easily integrated to give

$$
\xi=\frac{1}{y^{\prime 2} \mathrm{e}^{x}}\left[A \int y^{\prime} \mathrm{e}^{x} \mathrm{~d} x+B+\int y^{\prime} \mathrm{e}^{x}\left(\eta^{\prime \prime}+\eta^{\prime}+\eta y\right) \mathrm{d} x\right]
$$

In addition to the three symmetries of (3.24) we have the general symmetry

$$
G=\int\left\{\frac{1}{y^{\prime 2} \mathrm{e}^{x}}\left[\int y^{\prime} \mathrm{e}^{x}\left(\eta^{\prime \prime}+\eta^{\prime}+\eta y\right) \mathrm{d} x\right]\right\} \partial_{x}+\eta \partial_{y}
$$

for arbitrary functions $\eta$. If we apply the third extension of (3.28) to the equation (3.20), we obtain, after some simplification,

$$
\eta y^{\prime} h^{\prime}+\eta^{\prime} h=(\eta y)^{\prime}-\eta^{\prime},
$$

in which the prime on $\eta$ represents differentiation with respect to $x$ and the prime on $h(y)$ differentiation with respect to $y$. If we make use of the chain rule, we may write (3.29) as

$$
(\eta h)^{\prime}=(\eta y)^{\prime}-\eta^{\prime},
$$

where now all primes represent differentiation with respect to $y$ and it is evident that $\eta$ must be a function of $y$ only. We integrate (3.30) to obtain

$$
h(y)=\frac{A}{\eta}+y-1
$$

and so, up to a constant, we obtain (2.1) if we take $\eta$ to be a constant. 


\section{Reconstruction of the model equation}

The third order equation

$$
y^{\prime \prime \prime}+y^{\prime \prime}+K y^{\prime}+y y^{\prime}=0
$$

has the four symmetries

$$
\begin{gathered}
G_{1}=\partial_{x}, \quad G_{2}=\left(\int \frac{\mathrm{d} x}{y^{\prime 2} \mathrm{e}^{x}}\right) \partial_{x}, \\
G_{3}=\left(\int \frac{\mathrm{d} x}{y^{\prime 2}}\left(y-\mathrm{e}^{-x} \int y \mathrm{e}^{x} \mathrm{~d} x\right)\right) \partial_{x}, \\
G_{4}=\int\left\{\frac{1}{y^{\prime 2} \mathrm{e}^{x}}\left[\int y y^{\prime} \mathrm{e}^{x} \mathrm{~d} x\right]\right\} \partial_{x}+\partial_{y}
\end{gathered}
$$

which specify it completely. This, of course, is not the model equation with which we started and we would do well to verify that the presence of the additional term makes no difference to the behaviour of the solution of the equation.

When we perform the Painlevé analysis we find that the first and fourth terms in (4.1) are dominant, the singularity is a double pole and the resonances are $r=-1,4$ and 6 as for the model equation, (2.1). When we make the substitution

$$
y=\sum_{i=0}^{6} a_{i} \chi^{i-2}
$$

to check for compatibility, we find that

$$
a_{0}=-12, \quad a_{1}=\frac{12}{5}, \quad a_{2}=\frac{6}{25}-K, \quad a_{3}=\frac{6}{125},
$$

but then, at the resonance $r=4, a_{4}=0$ and so there can be no Painlevé property for (4.1).

Equation (4.1) is trivially integrated to give

$$
y^{\prime \prime}+y^{\prime}+K y+\frac{1}{2} y^{2}-I=0
$$

where $I$ is the value of the first integral. When we perform the Painlevé analysis on (4.5), we find that the singularity is a double pole and the resonances are at $r=-1$ and 6 . We make the same substitution as in (4.3) and find that the coefficients are given by

$$
\begin{gathered}
a_{0}=-12, \quad a_{1}=\frac{12}{5}, \quad a_{2}=\frac{1}{25}-K, \quad a_{3}=\frac{1}{125}, \\
a_{4}=\frac{7}{2500}-\frac{2 I+K^{2}}{20}, \quad a_{5}=\frac{158}{25000}-\frac{11\left(2 I+(K+1)^{2}\right)}{300},
\end{gathered}
$$

that $a_{6}$ is arbitrary and that there is compatibility at this resonance provided

$$
2 I+K^{2}=-\frac{36}{625}
$$


Finally we compute the Lie point symmetries of $(4.5)$ when the constants are related according to (4.7). They are

$$
G_{1}=\partial_{x} \quad \text { and } \quad G_{2}=\mathrm{e}^{x / 5}\left[\partial_{x}+\frac{2}{5}\left(\frac{6}{25}-K-y\right) \partial_{y}\right] .
$$

We observe that these features are identical to those of (2.1) when we make the substitution $K=0$. It is not feasible to make numerical experiments for arbitrary $K$, but we are confident that the behaviour of the solutions of (4.1) is the same as the solutions of our original model equation.

We can demonstrate a basis for that confidence by using the symmetries to reduce the order of (4.1). Naturally we commence with the only point symmetry, $G_{1}$, which has the invariants $u=y$ and $v=y^{\prime}$. Equation (4.5) becomes

$$
v v^{\prime \prime}+v^{\prime 2}+v^{\prime}+K+u=0
$$

and the other symmetries become

$$
\begin{gathered}
G_{2} \rightarrow\left(\frac{1}{v} \exp \left[-\int \frac{\mathrm{d} u}{v}\right]\right) \partial_{v}, \\
G_{3} \rightarrow\left(\frac{1}{v} \exp \left[-\int \frac{\mathrm{d} u}{v}\right] \int \exp \left[\int \frac{\mathrm{d} u}{v} \mathrm{~d} u\right]\right) \partial_{v}, \\
G_{4} \rightarrow \partial_{u}-\left(\frac{1}{v} \exp \left[-\int \frac{\mathrm{d} u}{v}\right] \int u \exp \left[\int \frac{\mathrm{d} u}{v} \mathrm{~d} u\right]\right) \partial_{v} .
\end{gathered}
$$

We observe that $G_{2}$ has become an exponential nonlocal symmetry and consequently can be used to reduce the order of (4.9) [5]. We obtain the invariants $z=u$ and $w=v v^{\prime}+v$ and (4.9) is reduced to the first order equation

$$
\frac{\mathrm{d} w}{\mathrm{~d} z}=-(K+z)
$$

We can now see that the presence of $K$ has no bearing upon the behaviour of the solution of the equation except as a translation of the initial condition on $y\left(x_{0}\right)$, thereby justifying our comment above. Under this reduction of order the two unused symmetries become

$$
G_{3} \rightarrow \partial_{w}, \quad G_{4} \rightarrow z \partial_{w}
$$

and so we can infer that these two symmetries have the Abelian algebra $2 A_{1}$.

As (4.11) has two Lie point symmetries, it is obviously integrable. We obtain

$$
w(z)=C-\frac{1}{2}(K+z)^{2}
$$

so that the next equation to be solved is

$$
v v^{\prime}+v=C-\frac{1}{2}(K+u)^{2}
$$

Equation (4.14) is an Abel's equation of the second kind which, not surprisingly, does not yield to any of the methods of solution proposed by Kamke [11, p. 24, Art 4.10 and 4.11]. 
In the integrable case the second order equation to be solved is

$$
y^{\prime \prime}+y^{\prime}+\frac{1}{2}(y+K)^{2}-\frac{18}{625}=0 .
$$

This can be transformed to an equation of Emden-Fowler type

$$
\ddot{w}+w^{2}=0
$$

by means of the Kummer-Liouville transformation

$$
y=2 \mathrm{e}^{2 x / 5} w(t)-K-\frac{6}{25}, \quad t^{\prime}(x)=\mathrm{e}^{-x / 5} .
$$

The solution of (4.16) is in terms of elliptic functions and so the solution of (4.15) is in terms of elliptic functions of somewhat complicated argument. It is interesting that on the surface in the space of initial conditions specified by the value of the integral this solution is analytic except for the polelike singularity and yet just off this surface one may find nonintegrable behaviour.

We can always make use of a Kummer-Liouville transformation to bring (4.5) into the form of a generalised Emden-Fowler equation. If we put

$$
y+K=u(x) v(t)+\alpha \quad \text { and } \quad t=t(x),
$$

(4.5) becomes

$$
\begin{gathered}
\left(u t^{\prime 2}\right) \ddot{v}+\left(2 u^{\prime} t^{\prime}+u t^{\prime \prime}+u t^{\prime}\right) \dot{v} \\
+\left(u^{\prime \prime}+u^{\prime}+u \alpha\right) v+\frac{1}{2} u^{2} v^{2}+\frac{1}{2} \alpha^{2}-\frac{1}{2} \beta^{2}=0,
\end{gathered}
$$

where we have written $2 I+K^{2}=\beta^{2}$. We remove the constant term by setting $\alpha=\beta$. We remove the coefficient of $v$ by solving the equation

$$
u^{\prime \prime}+u^{\prime}+u \beta=0
$$

The coefficient of $\dot{v}$ is removed if we set $t^{\prime}=u^{-2} \mathrm{e}^{-x}$ and we are left with the equation

$$
\ddot{v}+\phi(t) v^{2}=0
$$

where

$$
\phi(t(x))=\frac{1}{2} \mathrm{e}^{-x / 2}\left(A \mathrm{e}^{\gamma x}+B \mathrm{e}^{-\gamma x}\right)^{5},
$$

$A$ and $B$ are constants of integration and $\gamma=\sqrt{\frac{1}{4}-\left(2 I+K^{2}\right)^{1 / 2}}$. We note that the form of $\phi(t(x))$ is not one of the functions for which the generalised Emden-Fowler equation (4.21), has been found to be integrable [14, 21]. 


\section{Discussion}

As in the case of Krause's analysis for the complete symmetry group of the Kepler problem, we can specify our model equation up to a constant. The Kepler problem, being a threedimensional system of second order equations, required eight symmetries, three of which were nonlocal. Our scalar third order equation is similarly specified with four symmetries, three of which are nonlocal.

Already in the paper by Krause [12] there was some concern as to the proper way to consider the algebraic properties of the symmetries, particularly the nonlocal symmetries. In fact he comments "these are not point transformations and one does not obtain from them a set of differential operators satisfying a Lie algebra, as in the standard manner." [12, p. 5738]. The method used by Krause to overcome this obstacle was to look at the transformations generated by the symmetries in the coordinates and the rate of change of local time. In both of these the nonlocality of the symmetries did not appear and he was able to construct a satisfactory group representation.

Our approach in this matter is somewhat different because we attempt to obtain a representation of an algebra from the symmetries under consideration and consequently we do not want to have the ambiguities of the algebras of nonlocal symmetries to intrude as an unnecessary complicating factor. Our aim is to present the concept in the simplest possible context. We also consider the question of the uniqueness of the complete symmetry group, because we do not believe that it is unique. Rather one would expect there to be equivalent representations and we demonstrate that with the great paradigm, the one-dimensional free particle. The free particle in one dimension with the equation

$$
\ddot{x}=0
$$

has the eight Lie point symmetries

$$
\begin{array}{llll}
G_{1}=\partial_{x}, & G_{2}=t, \partial_{x}, & G_{3}=x \partial_{x}, & G_{4}=\partial_{t}, \\
G_{5}=2 t \partial_{t}+x \partial_{x}, & G_{6}=t^{2} \partial_{t}+t x \partial_{x}, & G_{7}=x \partial_{t}, & G_{8}=t x \partial_{t}+x^{2} \partial_{x}
\end{array}
$$

with the algebra $\operatorname{sl}(3, R)$ which has the substructure $\operatorname{sl}(2, R) \oplus_{s}\left\{2 A_{1} \oplus A_{2} \oplus A_{1}\right\}$. If we assume the general form of a second order equation,

$$
\ddot{x}=f(t, x, \dot{x}),
$$

we find, for example, that $f$ must take the value in (5.1) with the combinations of symmetries $G_{1}, G_{2}$ and $G_{3} ; G_{3}, G_{7}$ and $G_{8}$. The algebra of the each triple is $A_{3,3}$ ( $\Leftrightarrow D \oplus_{s} T_{2}$, the semidirect sum of dilations and translations). The triple $G_{1}, G_{7}$ and $G_{8}$ also completely specifies the equation, but it does not close. To obtain a closed algebra one must add the symmetries $G_{3}, G_{4}$ and $G_{5}$. The triple $G_{4}, G_{5}$ and $G_{6}$, constituting the subalgebra $s l(2, R)$ and characteristic of all differential equations of maximal symmetry, gives the Ermakov-Pinney equation

$$
\ddot{x}=\frac{K}{x^{3}}
$$

and needs an additional symmetry, say $G_{1}$, to recover (5.1). 
This example demonstrates quite clearly the possible nonuniqueness of the complete symmetry group since these three algebras are quite distinct and cannot be different realisations of the same group because they are of different dimension.

We conclude that we have demonstrated that even an ordinary differential equation which has Painlevé properties consistent with nonintegrable behaviour is defined in terms of its symmetries. We do not believe that our model equation, (2.1), is exceptional and propose that all ordinary differential equations, be they derived in a Newtonian or relativistic context or elsewhere, can be defined in terms of a set of Lie symmetries. We repeat the point made earlier at the conclusion of Section 2. The Lie symmetries of a differential equation both provide a means to identify the specific structure of the equation and a possible route towards its solution. The second property is not automatically available in any given symmetry. For a symmetry to be part of the route to the solution of a differential equation the symmetry must be able to provide a means for the reduction of order, if not immediately, at some level in the reduction process.

\section{Acknowledgements}

MCN thanks the MURST (Cofin 97: Metodi e applicazioni di equazioni differenziali ordinarie) for its support and PGLL expresses his deep appreciation of the hospitality of the Dipartimento di Matematica e Informatica, Università di Perugia, during the period in which this work was initiated, thanks the Dean of the School of Sciences, University of the Aegean, Professor G P Flessas, and the Director of GEODYSYC, Dr S Cotsakis, for their

kind hospitality while this work was undertaken and the National Research Foundation of South Africa and the University of Natal for their continuing support.

\section{References}

[1] Chang Y F, Tabor M and Weiss J, Analytic Structure of the Hénon-Heiles Hamiltonian in Integrable and Nonintegrable Regimes, J. Math. Phys. 23 (1982), 531-538.

[2] Cheb-Terrab E S and Roche A D, Integrating Factors for Second Order ODEs, J. Symb. Comp. 27 (1999), 501-519.

[3] Cotsakis S and Leach P G L, Painlevé Analysis of the Mixmaster Universe, J. Phys. A: Math. Gen. 27 (1994), 1625-1631.

[4] Dyer C C, McVittie G D and Oattes L M, A Class of Spherically Symmetric Solutions with Conformal Killing Vectors, Gen. Rel. Grav. 19 (1987), 887-898.

[5] Géronimi C, Feix M R and Leach P G L, Exponential Nonlocal Symmetries and Nonnormal Reduction of Order, Preprint: MAPMO, Département mathématique, Université d'Orléans, Orléans la Source, 45067 Orléans Cedex 2, France, 1997

[6] Gorringe V M and Leach P G L, Kepler's Third Law and the Oscillator's Isochronism, Amer. J. Phys. 61 (1993), 991-995.

[7] Govinder K S and Leach P G L, On the Determination of Nonlocal Symmetries, J. Phys. A: Math. Gen. 28 (1995), 5349-5359.

[8] Hall L S, A Theory of Exact and Approximate Configurational Invariants, Physica 8D (1983), $90-105$. 
[9] Head A K, LIE, a PC Program for Lie Analysis of Differential Equations, Comp. Phys. Commun. 77 (1993), 241-248.

[10] Ince E L, Ordinary Differential Equations, Longmans, Green \& Co - London, 1927.

[11] Kamke E, Differentialgleichungen Lösungsmethoden und Lösungen, B G Teubner - Stuttgart, 1983.

[12] Krause J, On the Complete Symmetry Group of the Classical Kepler System, J. Math. Phys. 35 (1994), 5734-5748.

[13] Kummer E E, De generali quadam æquatione differentiali tertii ordinis, J. für Math. 100 (1887), 1-9.

[14] Leach P G L, An Exact Invariant for a Class of Time-Dependent Anharmonic Oscillators with Cubic Anharmonicity, J. Math. Phys. 22 (1981), 465-470.

[15] Leach P G L and Bouquet S É, Symmetry, Singularities and Integrability in Complex Dynamics VI: Integrating Factors and Symmetries, Preprint, GEODYSYC, Department of Mathematics, University of the Aegean, Karlovassi 83 200, Greece, June, 2000.

[16] Leach P G L, Cotsakis S and Flessas G P, Symmetry, Singularities and Integrability in Complex Dynamical Systems I: The Reduction Problem, J. Nonlin. Math. Phys. 7 (2000), $445-479$.

[17] Leach P G L, Cotsakis S and Flessas G P, Symmetry, Singularities and Integrability in Complex Dynamical Systems II: Rescaling and Time-Translation in Two-Dimensional Systems, $J$. Math. Anal. Appl. 251 (2000), 587-608.

[18] Leach P G L, Moyo S, Cotsakis S and Lemmer R L, Symmetry, Singularities and Integrability in Complex Dynamics III: Approximate Symmetries and Invariants, J. Nonlin. Math. Phys. 8 (2001), 139-156.

[19] Lie S, Differentialgleichungen, Chelsea - New York, 1967.

[20] Liouville J, Sur le développement des fonctions ou parties des fonctions en séries dont les divers termes sont assujettis é satisfaire é une même équatione différentielle du second ordre contenent un paramètre variable, J. Math. pures appl. II (1837), 16-35.

[21] Mellin C M, Mahomed F M and Leach P G L, Solution of Generalised Emden-Fowler Equations with Two Symmetries, Int. J. Nonlin. Mech. 29 (1994), 529-538.

[22] Miritzis J, Leach P G L and Cotsakis S, Symmetry, Singularities and Integrabily in Complex Dynamical Systems IV: Painlevé Integrability of Isotropic Cosmologies, Grav. Cosm. 6 (2000), 291-302.

[23] Nucci M C, The complete Kepler group can be derived by Lie group analysis, J. Math. Phys. 37 (1996), 1772-1775.

[24] Nucci M C and Leach P G L, The Harmony in the Kepler and Related Problems, J. Math. Phys. 42 (2000), 746-764.

[25] Pillay T and Leach P G L, A General Approach to the Symmetries of Differential Equations, Prob. Nonlin. Anal. Eng. Sys. 2 (1997), 33-39.

[26] Sarlet W, Leach P G L and Cantrijn F, Exact Versus Configurational Invariants and a Weak Form of Complete Integrability, Physica 17D (1985), 87-98. 
[27] Sherring J, Head A K and Prince G E, Dimsym and LIE: Symmetry Determining Packages, Math. Comp. Model. 25 (1997), 153-164.

[28] Tabor M, Chaos and Integrability in Nonlinear Dynamics, John Wiley \& Sons - New York, 1989. 